\title{
Galactic Wind in the Nearby Starburst Galaxy NGC 253 Observed with the Kyoto3DII Fabry-Perot Mode
}

\author{
K. Matsubayashi ${ }^{1}$, H. Sugai ${ }^{1}$, T. Hattori ${ }^{2}$, A. Kawai ${ }^{1}$, S. Ozaki ${ }^{3}$, G. Kosugi ${ }^{4}$, T. Ishigaki ${ }^{5}$,
} A. Shimono ${ }^{1}$

\begin{abstract}
We have observed the central region of the nearby starburst galaxy NGC 253 with the Kyoto Tridimensional Spectrograph II (Kyoto3DII) Fabry-Perot mode in order to investigate the properties of its galactic wind. Since this galaxy has a large inclination, it is easy to observe its galactic wind. We produced the $\mathrm{H} \alpha$, $[\mathrm{N}$ II] $\lambda 6583$, and $[\mathrm{S} \mathrm{II}] \lambda \lambda 6716,6731$ images, as well as those line ratio maps. The $[\mathrm{N} \mathrm{II}] / \mathrm{H} \alpha$ ratio in the galactic wind region is larger than those in $\mathrm{H}$ II regions in the galactic disk. The $[\mathrm{N} \mathrm{II}] / \mathrm{H} \alpha$ ratio in the southeastern filament, a part of the galactic wind, is the largest and reaches about 1.5. These large $[\mathrm{N} \mathrm{II}] / \mathrm{H} \alpha$ ratios are explained by shock ionization/excitation. Using the $[\mathrm{S} \mathrm{II}] / \mathrm{H} \alpha$ ratio map, we spatially separate the galactic wind region from the starburst region. The kinetic energy of the galactic wind can be sufficiently supplied by supernovae in a starburst region in the galactic center. The shape of the galactic wind and the line ratio maps are non-axisymmetric about the galactic minor axis, which is also seen in M82. In the $[\mathrm{N}$ II $] \lambda 6583 /[\mathrm{S} \mathrm{II}] \lambda \lambda 6716,6731$ map, the positions with large ratios coincide with the positions of star clusters found in the Hubble Space Telescope (HST) observation. This means that intense star formation causes strong nitrogen enrichment in these regions. Our unique data of the line ratio maps including [S II] lines have demonstrated their effectiveness for clearly distinguishing between shocked gas regions and starburst regions, determining the extent of galactic wind and its mass and kinetic energy, and discovering regions with enhanced nitrogen abundance.
\end{abstract}

\footnotetext{
${ }^{1}$ Department of Astronomy, Kyoto University, Sakyo-ku, Kyoto 606-8502, Japan; kazuya@kusastro.kyotou.ac.jp

${ }^{2}$ Subaru Telescope, National Astronomical Observatory of Japan, 650 North A'ohoku Place, Hilo, Hawaii 96720, USA

${ }^{3}$ Okayama Astrophysical Observatory, National Astronomical Observatory of Japan, Kamogata, Okayama 719-0232, Japan

${ }^{4}$ National Astronomical Observatory of Japan, Mitaka, Tokyo 181-8588, Japan

${ }^{5}$ Asahikawa National College of Technology, Asahikawa, Hokkaido 071-8142, Japan
} 
Subject headings: galaxies: individual(NGC 253) — galaxies: starburst — ISM: abundances — ISM: jets and outflows

\section{INTRODUCTION}

Galactic scale outflows (galactic winds) from galactic disks are common phenomena in the universe: they are found both in nearby galaxies (e.g., Strickland et al. 2004; Tüllmann et al. 2006) and in high-z galaxies (e.g., Shapley et al. 2003; Tapken et al. 2007). Their main sources of energy are stellar winds, supernovae, or active galactic nuclei (AGNs). Their asymmetric and complex structures (e.g., M82; Shopbell \& Bland-Hawthorn 1998, NGC 253; Sugai et al. 2003) are investigated by numerical simulations (e.g., Cooper et al. 2008). Galactic winds are considered to impact on galaxies and their environments. Kobayashi et al. (2007) takes galaxy-scale outflows into their semi-analytical model of galaxy formation as a parameter, which reproduces the observed Ly $\alpha$ luminosity functions of Ly $\alpha$ emitters in high-z. Recently, Sato et al. (2008) has suggested from their multi-wavelength observation data that galactic winds may carry away interstellar gas in host galaxies and may quench star formation. Galactic winds from dwarf galaxies with gas masses of a few $\times 10^{8} M_{\odot}$ are suggested to be the major pollutants of the intergalactic medium (IGM) through an analytical/numerical model (Ferrara \& Tolstoy 2000). Veilleux et al. (2005) and Bland-Hawthorn et al. (2007), in reviews of the galactic winds, summarize the properties and significance of galactic winds.

Despite the importance of galactic winds, there are few galaxies where the basic physical values (e.g., mass and kinetic energy) of the galactic winds were derived from spatially resolved data. It is important to analyse spatially resolved data because galactic winds have extremely complex structures: e.g., non-axisymmetry about the minor axis of a galactic disk is found in M82 (Shopbell \& Bland-Hawthorn 1998) and NGC 253 (Sugai et al. 2003). The Fabry-Perot Interferometer, which enables us to subtract the continuum precisely and to produce pure line images, is a powerful method to observe galactic winds. The galactic winds in M82 (Shopbell \& Bland-Hawthorn 1998) and NGC 1482 (Veilleux \& Rupke 2002) were observed with Fabry-Perot instruments, and their masses, kinetic energies, and excitation sources of them were estimated from the $[\mathrm{N} \mathrm{II}] \lambda 6583 / \mathrm{H} \alpha$ line ratio maps. In spite of the fact that this method is especially effective, there were only three examples where the properties of galactic winds in edge-on galaxies were discussed in detail from line ratio maps.

NGC 253 is one of the most famous galaxies because it is a nearby $(\mathrm{D}=3.5 \mathrm{Mpc}$ : Rekola et al. 2005) edge-on (inclination $=78^{\circ}$ : Pence 1980) starburst galaxy. Details of this galaxy have been investigated in many wavelengths such as X-ray (Strickland et al. 2000), 
optical (Schulz \& Wegner 1992; Watson et al. 1996), infrared (Sams et al. 1994; Sugai et al. 2003), sub-mm (Sakamoto et al. 2006), and radio (Turner \& Ho 1985; Ulvestad \& Antonucci 1997). In NGC 253, the galactic wind was found in $\mathrm{H} \alpha$ (e.g., McCarthy et al. 1987; Schulz \& Wegner 1992) and in X-ray (e.g., Strickland et al. 2000), and the properties of the galactic wind were discussed in them. The expansion velocity is estimated through slit spectroscopic observation in optical (Schulz \& Wegner 1992). Strickland et al. (2000) discuss the spatial structure of the outflow from the azimuthal surface brightness profiles of the X-ray and the $\mathrm{H} \alpha$.

We have observed the central region of NGC 253 with the Fabry-Perot(FP) mode of the Kyoto Tridimensional Spectrograph II (Kyoto3DII: Sugai et al. 2000, 2002, 2004) mounted on the Subaru Telescope. The Kyoto3DII is a unique instrument that enables us to carry out the optical Fabry-Perot observations on an 8-meter class telescope and to observe weak emission lines. The observations were carried out during the test observations of the Kyoto3DII, and this is the first result of the Kyoto3DII FP mode. From these data, we have obtained the $[\mathrm{N} \mathrm{II}] \lambda 6583 / \mathrm{H} \alpha$, [S II $] \lambda \lambda 6716,6731 / \mathrm{H} \alpha$, and $[\mathrm{N} \mathrm{II}] /[\mathrm{S}$ II] line ratio maps. This unique set of line ratio maps allows us to clearly distinguish between shocked gas regions and starburst regions, to determine the extent of galactic wind as well as its mass and kinetic energy, and to discover regions with enhanced nitrogen abundance.

We describe our observations and data reduction in Section 2. We show the $\mathrm{H} \alpha$ and continuum images and the line ratio maps, and compare our images with the X-ray and the radio images in Section 3. We discuss the properties of the galactic wind and stellar clusters from the continuum and $\mathrm{H} \alpha$ images and the line ratio maps in Section 4 . We summarize our conclusions in Section 5 .

\section{OBSERVATIONS AND DATA REDUCTION}

We have observed the central region of NGC 253 with the Fabry-Perot(FP) mode of the Kyoto3DII (Sugai et al. 2000, 2002, 2004) on 28 August 2002 during the test observation of the Kyoto3DII. It was mounted on the Cassegrain focus of the Subaru Telescope at Mauna Kea. This mode uses an ET-50 etalon made by Queensgate Instruments. The field of view is $\sim 1^{\prime} .9 \times 1^{\prime} .9$, with the spatial sampling of $0^{\prime \prime} .112$ after $2 \times 2$ on-chip binning. The spatial resolution was $1^{\prime \prime} .0$ and the spectral resolution was $21 \AA$. For each of the $\mathrm{H} \alpha+[\mathrm{N} \mathrm{II}] \lambda \lambda 6548,6583$ and [S II] $\lambda \lambda 6716,6731$ sets, we obtained 5 on-band frames and 1 offband frame. The central wavelengths of the transmission curve of etalon for the $\mathrm{H} \alpha+[\mathrm{N}$ II] set were $6556 \AA, 6568 \AA, 6580 \AA, 6592 \AA, 6604 \AA$ (on-band), and $6502 \AA$ (off-band), while those of the $[\mathrm{S}$ II] $] \lambda \lambda 6716,6731$ set were $6717 \AA, 6729 \AA, 6741 \AA, 6754 \AA, 6766 \AA$ (on-band), and $6650 \AA$ (off-band). The exposure time of each frame was 240 seconds. The bias subtraction 
and the flat fielding have been performed for the target object frames and the standard star frames, and then the flux calibration has been done. For reducing the observing time, flat and standard star frames were not obtained at all the observed wavelengths. Therefore, we have used the flat frames of the nearest wavelength for the flat fielding and the interpolated data for the flux calibration. The flat flames in $6502 \AA, 6568 \AA$, and $6650 \AA$ were similar to each other: $\sim 20 \%$ brighter at the frame center compared with at the edge. The flat flames in longer wavelengths, including in $6729 \AA, 6742 \AA, 6749 \AA$, and $6757 \AA$, had a different flat pattern: darker at the frame center. In the longest wavelength, it was $\sim 30 \%$ darker at the center. The resultant uncertainty caused by the flat fielding, including the interpolation, is negligible in shorter wavelengths, and within a few percent in longer wavelengths. We have removed cosmic rays, matched the spatial resolution to the worst frame, and subtracted the off-band frames from the on-band frames. Eventually, the frames including only emission lines were obtained. The sky subtraction has not been performed in each frame because objects are observed in the whole field of view. This affects the continuum image, but does not affect the line images because the sky emission has been subtracted through the process of the off-band subtraction.

Because of the low spectral resolution, the $\mathrm{H} \alpha$ and the [N II] $\lambda \lambda 6548,6583$ in the $\mathrm{H} \alpha+[\mathrm{N} \mathrm{II}]$ set, as well as the $[\mathrm{S} \mathrm{II}] \lambda \lambda 6716,6731$ in the $[\mathrm{S} \mathrm{II}]$ set, were blended. In order to decompose them, we have fitted these lines pixel by pixel with Airy functions, which are the transmission curve of the Fabry-Perot Interferometer (Bland \& Tully 1989). Figure 1 shows examples of the fitting. The residual ratios were calculated by dividing the total residuals by the total flux of the $\mathrm{H} \alpha$ plus [NII] $\lambda \lambda 6548,6583$, and they were $5 \%$ and $30 \%$ in good and poor examples, respectively. In poor fitting, line decomposing may be inaccurate, and the flux ratio may have a large error. However, the fraction of poor examples was smaller than 1/100, and much smaller in the regions we discuss below, and we neglected them. The average residual ratio for a single pixel in the discussed regions was $15 \%$. As free parameters, we took the fluxes of the $\mathrm{H} \alpha$ and $[\mathrm{N} \mathrm{II}] \lambda 6583$ in the $\mathrm{H} \alpha+[\mathrm{N}$ II] set, and those of the $[\mathrm{S} \mathrm{II}] \lambda 6716$ and [S II] $\lambda 6731$ in the $[\mathrm{S} \mathrm{II}]$ set. We assumed that the flux of the $[\mathrm{N} \mathrm{II}] \lambda 6548$ was one third of that of the $[\mathrm{N} \mathrm{II}] \lambda 6583$. For the fitting, we fixed the velocity center so that it corresponded to the heliocentric velocity of NGC 253 of $251 \mathrm{~km} \mathrm{~s}^{-1}$ (Watson et al. 1996). We also fixed the intrinsic velocity dispersion as zero because it was much smaller than the instrumental profile. The main source of the uncertainties for the line ratio maps is not flux calibration but line fitting, because the sky transmittance was stable during the observation for NGC 253. In order to estimate the uncertainties of the fitting, we considered pseudo-observation data: they were obtained with the same "observing" parameters as actual observations from the spectra which include maximum velocity variation, velocity dispersion, and line-splitting observed in slit-spectroscopy (Schulz \& Wegner 1992). The uncertainties were estimated 
from the difference between the ratio derived from the fitting and the input intrinsic ratio. The uncertainties of the $[\mathrm{N} \mathrm{II}] / \mathrm{H} \alpha$ and $[\mathrm{S} \mathrm{II}] / \mathrm{H} \alpha$ maps were smaller than $30 \%$ and $10 \%$, respectively. The uncertainty of the absolute flux calibration was less than $20 \%$, and the uncertainty of the $\mathrm{H} \alpha$ flux, including the line fitting, was 30\%. As for the [S II] $]$ 6716/[S II] $] 6731$ ratio, it can be largely affected by the velocity field. Therefore, the [S II] ratio map is not presented. There was a distortion aberration, as expected, in the frames and we have corrected it. The background noise level was $1.9 \times 10^{-16} \mathrm{erg} \mathrm{cm}^{-2} \mathrm{~s}^{-1} \operatorname{arcsec}^{-2}$ in the H $\alpha$ set and $6.3 \times 10^{-17} \mathrm{erg} \mathrm{cm}^{-2} \mathrm{~s}^{-1} \operatorname{arcsec}^{-2}$ in the [S II] set, respectively. We determined the absolute coordinate by comparing our FP continuum frame with the continuum frame of HST Wide-Field Planetary Camera 2 (WFPC2) (F675W: Watson et al. 1996). The uncertainty of the coordinate between the FP and the HST frames was estimated to be $0^{\prime \prime} .08(1 \sigma)$. We adopt a distance of 3.5 Mpc to NGC 253 (Rekola et al. 2005).

\section{RESULTS}

\subsection{The continuum and $\mathrm{H} \alpha$ images}

Figure 2 (a) shows the continuum image of central region of NGC 253. Figures 3 (a), 2 (b), and 2 (c) show the whole field of view, the central region (deep), and the central region (shallow) $\mathrm{H} \alpha$ images, respectively. The continuum image is obtained from the average flux of the two off-band frames. In the central region, two bright regions are found in the $\mathrm{H} \alpha$ and continuum emission. One is found at 4 "southwest from the center of NGC 253, while the other is found at $5^{\prime \prime}$ southeast from the center. Hereafter, we call the former starburst region $\mathrm{A}$, and the latter starburst region $\mathrm{B}$. A filamentary structure is seen to the northeast of the center and we call it filament $\mathrm{C}$. In regions farther from the center, there are two bright regions in the $\mathrm{H} \alpha$ image. One is found at $\sim 30^{\prime \prime}$ east from the center, and the other is found at $\sim 60^{\prime \prime}$ southwest from the center. We call the former $\mathrm{H}$ II region $\mathrm{A}$, and the latter $\mathrm{H}$ II region B. We analyse the characteristics of these regions in Section 3.2.

In the $\mathrm{H} \alpha$ and $[\mathrm{N} \mathrm{II}]$ images, two faint filaments are seen to the southeast of the center. They were found in the narrowband $\mathrm{H} \alpha+[\mathrm{N} \mathrm{II}]$ image (McCarthy et al. 1987: the H $\alpha$ and [N II] lines are blended in their image). These filamentary structures are observed at the edges of the outflow when we observe the conical shell-like optically-thin gas (e.g., Strickland et al. 2000; Sugai et al. 2003). The positions of these filamentary structures coincide with those in

the near-infrared $\mathrm{H}_{2}$ emission (features A and B: Sugai et al. 2003). Hereafter, we call the southwest one filament $\mathrm{A}$ and the southeast one filament B. We refer to these filamentary structures as "filaments", although we consider that they are not filaments but actually are a part of conical outflow. The north-side features were also found in the $\mathrm{H}_{2}$ emission, but 
not in the $\mathrm{H} \alpha$ probably due to strong extinction. The positions of filamentary structures seen in the $\mathrm{H} \alpha$ and in the soft X-ray (Strickland et al. 2000) in the galactic wind are close to each other (Figures $2(\mathrm{~h})$ and $3(\mathrm{~b}))$. This indicates that the relatively warm $\left(\sim 10^{4} \mathrm{~K}\right)$ gas exists closely to the hot $\left(\sim 10^{6} \mathrm{~K}\right)$ gas. A closer inspection, however, reveals that the $\mathrm{X}$-ray filaments tend to exist where the $\mathrm{H} \alpha$ emissions are locally weak and tend to exist in inner regions compared with the $\mathrm{H} \alpha$ filaments: the separation between the $\mathrm{H} \alpha$ and the $\mathrm{X}$-ray filaments is $\sim 70 \mathrm{pc}$. This represents that the inner part of the galactic wind has a higher temperature than the outer part of it. This relation between the spatial distributions of the $\mathrm{H} \alpha$ and the X-ray has been found through our accurate positioning of these two maps (cf. Strickland et al. 2000). The spatial resolution of our $\mathrm{H} \alpha$ image $\left(=1^{\prime \prime} .0\right)$ also is slightly better than that obtained by Strickland et al. $2000\left(=1^{\prime \prime} .5\right)$.

Starburst region B is as bright as starburst region A in the $\mathrm{H} \alpha$ and the continuum at $\sim 6600 \AA$, while the counterpart is not found in the near-infrared (Sams et al. 1994) or radio observations (Ulvestad \& Antonucci 1997). This region is diffuse in the optical broadband images retrieved from $H S T$ archives. A comparison of our $\mathrm{H} \alpha$ map and long-slit spectroscopic data (Schulz \& Wegner 1992) has revealed that starburst region B has the sharp local-minimum velocity of $-100 \mathrm{~km} \mathrm{~s}^{-1}$.

\subsection{Line ratio maps}

Figures 2 (d) and 2 (e) show the [N II] $\lambda 6583 / \mathrm{H} \alpha$ and [S II] $\lambda \lambda 6716,6731 / \mathrm{H} \alpha$ ratio maps, respectively. The $[\mathrm{N} \mathrm{II}] / \mathrm{H} \alpha$ line ratio varies widely among regions. The $[\mathrm{N} \mathrm{II}] / \mathrm{H} \alpha$ ratios of filaments A and B are larger than the other regions. The ratio is particularly the largest at the tip of filament $\mathrm{B}$ and reaches about 1.5. As leaving from the galactic center along the minor axis, the $\mathrm{H} \alpha$ flux of the galactic wind becomes weaker while its $[\mathrm{N} \mathrm{II}] / \mathrm{H} \alpha$ ratio tends to become larger. The $[\mathrm{N} \mathrm{II}] / \mathrm{H} \alpha$ ratios of starburst regions $\mathrm{A}$ and $\mathrm{B}$ are $\sim 0.5$, which are local minima in the central region and the galactic wind. The $[\mathrm{N} \mathrm{II}] / \mathrm{H} \alpha$ ratios of $\mathrm{H}$ II regions $\mathrm{A}$ and $\mathrm{B}$ are $\sim 0.25$ and are smaller than in the other regions. These ratios coincide with those of H II regions in nearby disk galaxies (Moustakas \& Kennicutt 2006). Therefore, we conclude that these regions are H II regions, which is why we call them H II regions A and B. As for H II region B, McCarthy et al. (1987) observed a part of this region and suggested that it is an $\mathrm{H}$ II region. Our data show fairly uniform distribution of the $[\mathrm{N} \mathrm{II}] / \mathrm{H} \alpha$ ratio of $\sim 0.25$ over the whole $\mathrm{H}$ II region $\mathrm{B}$ including its three clumps. The $[\mathrm{N} \mathrm{II}] / \mathrm{H} \alpha$ ratio of filament $\mathrm{C}$ is similar to those of $\mathrm{H}$ II regions $\mathrm{A}$ and $\mathrm{B}$, rather than those of the galactic wind or starburst regions $\mathrm{A}$ and $\mathrm{B}$. Therefore, this region is a star-forming region located on the same line of sight as an edge of the galactic wind although the region looks as if it is a part 
of the galactic wind. In Figure 2 (i) we compare our $\mathrm{H} \alpha$ map with the $\mathrm{CO}$ intensity map (Paglione et al. 2004). We find that the position of filament $\mathrm{C}$ coincides with that of a strong CO emission elongated along the $x_{2}$ orbit (Binney et al. 1991; Athanassoula 1992), which is made by and is oriented perpendicular to the bar (Peng et al. 1996; Paglione et al. 2004). Hence, star formation in filament $\mathrm{C}$ occurs due to this $x_{2}$ orbit. The $[\mathrm{S} \mathrm{II}] \lambda \lambda 6716,6731 / \mathrm{H} \alpha$ ratio map is globally similar to the $[\mathrm{N} \mathrm{II}] / \mathrm{H} \alpha$ ratio map: e.g., the $[\mathrm{S} \mathrm{II}] / \mathrm{H} \alpha$ ratios of filaments $\mathrm{A}$ and $\mathrm{B}$ are the largest among regions while those of $\mathrm{H}$ II regions $\mathrm{A}$ and $\mathrm{B}$ are the smallest. The $[\mathrm{S} \mathrm{II}] / \mathrm{H} \alpha$ ratios remain large outside of the filaments while the $[\mathrm{N} \mathrm{II}] / \mathrm{H} \alpha$ ratios become smaller. This may be because the metallicity of the galactic winds is larger than that outside of the filaments (see Section 4.2).

Figure 2 (f) shows the $[\mathrm{N}$ II] $\lambda 6583 /[\mathrm{S}$ II] $\lambda \lambda 6716,6731$ ratio map. The $[\mathrm{N}$ II]/[S II] ratios in the central region and the galactic wind are almost uniform and are $\sim 1.5$, while the $\mathrm{H} \alpha$ fluxes vary. The ratio of filament $\mathrm{C}$ is small and is $\sim 1.0$. Two large $[\mathrm{N} \mathrm{II}] /[\mathrm{S}$ II $]$ regions, whose ratios are greater than 2.0, are found to the north of starburst region A (Figure 4). Their positions coincide with those of star clusters found in HST observation (the bright blob and spot i: Watson et al. 1996). The cause of the large [N II]/[S II] ratio is probably the nitrogen enrichment due to star clusters. We will discuss this in detail in Section 4.2.

Figure 2 (g) shows the $\mathrm{H} \alpha$ equivalent width map calculated from the $\mathrm{H} \alpha$ and continuum images. The equivalent width of H II region A is $95 \AA$ on average and larger than $150 \AA$ at its center, which is the largest among regions. Starburst regions A and B have local maxima of the equivalent width in the central region: $88 \AA$ and $64 \AA$, respectively. Three clumps of $\mathrm{H}$ II region $\mathrm{B}$ have about the same equivalent widths with each other, ranging from $36 \AA$ to $39 \AA$. When the underlying diffuse continuum emission is subtracted, the equivalent widths of H II regions $\mathrm{A}$ and $\mathrm{B}$ are $~ 300-500 \AA$ (Table 1). As diffuse continuum emission regions, we selected regions that are near the H II regions and do not include extra emission. This estimation of the $\mathrm{H} \alpha$ equivalent widths has an uncertainty of a factor 2 at most, mainly caused by the uncertainty of determination of this diffuse continuum level.

The aperture, the $\mathrm{H} \alpha$ flux, the line ratios, and the equivalent width of each region are listed in Table 1, The star formation rate is estimated from the $\mathrm{H} \alpha$ flux and also is listed in the table. As an example, we adopted the $6^{\prime \prime} .0$ diameter circular aperture in measuring values of $\mathrm{H}$ II region A. The measured $\mathrm{H} \alpha$ flux of $\mathrm{H}$ II region A is $3.5 \times 10^{-13} \mathrm{erg} \mathrm{cm}^{-2}$ $\mathrm{s}^{-1}$, and its $\mathrm{H} \alpha$ luminosity is $5.1 \times 10^{38} \mathrm{erg} \mathrm{s}^{-1}$. From this $\mathrm{H} \alpha$ luminosity, the number of ionizing photons is estimated to be $3.7 \times 10^{50} \mathrm{~s}^{-1}$ and the star formation rate is estimated to be $4.0 \times 10^{-3} M_{\odot} \mathrm{yr}^{-1}$ (Kennicutt 1998). 


\section{DISCUSSION}

\subsection{Filament B}

At the tip of filament $\mathrm{B}$, the $[\mathrm{N} \mathrm{II}] / \mathrm{H} \alpha$ ratio reaches about 1.5 (see Section $[3.2$ ). An ordinary photoionization process in H II regions cannot produce this large $[\mathrm{N} \mathrm{II}] / \mathrm{H} \alpha$ ratio of filament B, while shock ionization/excitation is plausible. Although Schulz \& Wegner (1992) produced the $[\mathrm{N} \mathrm{II}] / \mathrm{H} \alpha$ ratio larger than 1.0 with photoionization model, this model used a single stellar temperature for ionizing stars. The photoionization model by Kewlev et al. (2001) is more reliable, because they took into consideration stellar population. Kewley et al. (2001) placed a theoretical upper limit for starburst models on the optical diagnostic diagrams (their equation (5) and Figure 16(a)), which show that it is difficult to exceed 1.0 in the $[\mathrm{N} \mathrm{II}] / \mathrm{H} \alpha$ ratio with starbursts even though they took into consideration the effects of the nitrogen enrichment. No combination of parameters, such as star formation history and ionization parameter, can generate a theoretical point above this fold. This limit is rather conservative, and Kauffmann et al. (2003) placed a demarcation line at lower [N II]/H $\alpha$ ratio from observational data. Photoionization may somewhat contribute near the central region, where the $[\mathrm{N} \mathrm{II}] / \mathrm{H} \alpha$ ratio is smaller than 1.0.

According to the shock model calculated by Dopita \& Sutherland (1995), the [N II]/H $\alpha$ ratio reaches to 1.55 , in the case of the solar-abundance interstellar gas, the magnetic parameter $B / n^{1 / 2}$ of $2 \mu \mathrm{G} \mathrm{cm}{ }^{3 / 2}$, which is the intermediate value among their models, and

shock velocity of $500 \mathrm{~km} \mathrm{~s}^{-1}$. This model can explain the largest [N II] $/ \mathrm{H} \alpha$ ratio in our data, $\sim$ 1.5. By using the $[\mathrm{S} \mathrm{II}] \lambda \lambda 6716,6731 / \mathrm{H} \alpha$ ratio map and the $[\mathrm{N} \mathrm{II}] / \mathrm{H} \alpha$ ratio map, it is possible to discuss the possibility of nitrogen enrichment. Figure 5 shows the $[\mathrm{N}$ II] $/ \mathrm{H} \alpha$ ratio vs the $[\mathrm{S} \mathrm{II}] / \mathrm{H} \alpha$ ratio diagram of a shock model (shock-only: Dopita \& Sutherland 1995). The observed ratios (squares and triangles) plotted above the model curves indicate that the $\mathrm{N} / \mathrm{O}$ ratio is about 1.8 times the solar value when we assume that the magnetic parameter is equal to 2 and there is no contribution of photoionization for these ratios. We used the Anders \& Grevesse (1989) abundance set, because photoionization and shock models that we referred to (Kewley et al. 2001; Dopita \& Sutherland 1995) use it. If new abundance set (Grevesse et al. 2007) is used and if the model flux is proportional to the amount of each element, the N/O ratio will be 2.5 times the solar value. Both the $[\mathrm{N} \mathrm{II}] / \mathrm{H} \alpha$ and the $[\mathrm{S}$ II $] / \mathrm{H} \alpha$ ratios are larger in further positions from the galactic disk in filament $\mathrm{B}$. This means that the shock velocity or the degree of contribution of photoionization varies among these positions.

We compare the $[\mathrm{N} \mathrm{II}] / \mathrm{H} \alpha$ ratio of the galactic wind in NGC 253 with those in other galaxies. The $[\mathrm{N} \mathrm{II}] / \mathrm{H} \alpha$ ratio of the galactic wind in $\mathrm{M} 82$, which is one of the most famous 
edge-on galaxies, is mainly less than 1.0, and photoionization by starburst is considered to be an ionization/excitation source (Shopbell \& Bland-Hawthorn 1998). There may be an influence of shock since the $[\mathrm{N} \mathrm{II}] / \mathrm{H} \alpha$ ratio tends to increase far from the galactic disk. In NGC 1482, in contrast, the $[\mathrm{N} \mathrm{II}] / \mathrm{H} \alpha$ ratio is larger than 1.0 in the bulk of the galactic wind and reaches to 2.0 (Veilleux \& Rupke 2002). This indicates shock as the ionization/excitation source. The $[\mathrm{N} \mathrm{II}] / \mathrm{H} \alpha$ ratio of galactic wind in NGC 253 is between the ratios observed for galactic winds in M82 and NGC 1482. The typical [N II]/H $\alpha$ ratios are 0.3-0.6 in M82 (Shopbell \& Bland-Hawthorn 1998) and 1.0-2.3 in NGC 1482 (Veilleux \& Rupke 2002), while it is $0.8-1.5$ in NGC 253 .

The shape of galactic wind and the line ratio maps are non-axisymmetric about the galactic minor axis, which is also seen in M82 (Shopbell \& Bland-Hawthorn 1998). The non-axisymmetric structure in NGC 253 is also found in the $\mathrm{H}_{2}$ emission, which suggests the inhomogeneous nature of the interstellar medium (Sugai et al. 2003). Our [N II]/H $\alpha$ ratio map shows that filament B has a larger ratio than filament A. This may occur if filament A is more affected by photoionization compared with filament $\mathrm{B}$, or if the mass or the velocity of the wind gas varies from region to region.

\subsection{Nitrogen enrichment}

Starburst regions $\mathrm{A}$ and $\mathrm{B}$ emit the $\mathrm{H} \alpha$ and the continuum strongly and have low $[\mathrm{N}$ II $] / \mathrm{H} \alpha$ ratios. Their $[\mathrm{N}$ II $] / \mathrm{H} \alpha$ ratios are larger than those of $\mathrm{H}$ II regions in nearby disk galaxies (Moustakas \& Kennicutt 2006), and are about twice as large as those of H II regions A and B in NGC 253 itself (Figure 2 (d)). These large [N II]/H $\alpha$ ratios are probably caused by nitrogen enrichment, as discussed in the following. Nitrogen enrichment relative to oxygen in metal rich environment was found in a statistical work by Liang et al. (2006). The nitrogen enrichment can easily explain the observed large $[\mathrm{N} \mathrm{II}] / \mathrm{H} \alpha$ as well as $[\mathrm{N} \mathrm{II}] /[\mathrm{S}$ II] ratio although distorted ionizing continuum, which mimicks the situation where neutral matter lies between the ionizing stars and the emission-line clouds (Schulz \& Wegner 1992), or shock may also increase the $[\mathrm{N} \mathrm{II}] / \mathrm{H} \alpha$ ratio.

Nitrogen enrichment occurs in the following process (e.g., Pagel 1997; Sugai et al. 2004). There are two kinds of elements produced in stars, i.e., the primary element and the secondary element. The primary element, such as oxygen and sulfur, is produced from hydrogen and helium. The ratio of the produced amount of each element (e.g., sulfur to oxygen) is constant and insensitive to the metallicity at the star birth. On the other hand the secondary element, such as nitrogen, is produced also in the CNO cycle, and is spread into the interstellar medium with the stellar wind. The produced amount increases with an increase 
in the metallicity at the star birth. Therefore, in a metal rich environment, the ratio of the produced amount of nitrogen (secondary element) to that of sulfur (primary element) becomes larger, and the $[\mathrm{N}$ II $] /[\mathrm{S}$ II $]$ ratio becomes large. The relationship between the $[\mathrm{N} \mathrm{II}] /[\mathrm{S} \mathrm{II}]$ ratio and the metallicity of galaxies is found in SDSS observations (Liang et al. 2006).

In the $[\mathrm{N} \mathrm{II}] /[\mathrm{S} \mathrm{II}]$ ratio map (Figure 2 (f)), the $[\mathrm{N} \mathrm{II}] /[\mathrm{S} \mathrm{II}]$ ratios of starburst regions $\mathrm{A}$ and $\mathrm{B}$ are larger than those of $\mathrm{H}$ II regions $\mathrm{A}$ and $\mathrm{B}$ and filament $\mathrm{C}$. This indicates that starburst regions $\mathrm{A}$ and $\mathrm{B}$ have higher metallicity. The $[\mathrm{N} \mathrm{II}] /[\mathrm{S}$ II] ratios in the bright blob and spot i (hereafter, we call it blob/spot region) are the largest (Figure4), so that the degree of nitrogen enrichment is extremely high. The de-excitation of [S II] (Osterbrock \& Ferland 2006) may cause this large $[\mathrm{N}$ II $] /[\mathrm{S}$ II $]$ ratio. The electron densities are estimated to be no more than $\sim 900 \mathrm{~cm}^{-3}$ from $[\mathrm{S} \mathrm{II}] \lambda 6716 /[\mathrm{S} \mathrm{II}] \lambda 6731$ ratios observed by Schulz \& Wegner (1992). This ratio is at the minimum $(\sim 0.9)$ around the blob/spot region in the whole field of view of their spectroscopic observations. These electron densities are small and the de-excitation reduces the $[\mathrm{S} \mathrm{II}]$ flux by no more than $16 \%$. The bright blob and spot i are bright in J, H, and K band (Sams et al. 1994), and are two out of four clusters defined in the optical continuum in HST observation (Watson et al. 1996). The bright blob is the brightest cluster among the four. Spot $\mathrm{i}$ is the second brightest in the $\mathrm{H} \alpha$ flux. We estimate the metallicity of blob/spot region from the $[\mathrm{N} \mathrm{II}] /[\mathrm{S} \mathrm{II}]$ ratio, 2.19. This ratio is at the largest end of the distribution for starburst galaxies analyzed by Liang et al. (2006). Assuming the relation between the $[\mathrm{N} \mathrm{II}] /[\mathrm{S} \mathrm{II}]$ ratio and the metallicity in starburst galaxies (Figure $3(\mathrm{~d}$ ) by Liang et al. 2006), the metallicity $(12+\log (\mathrm{O} / \mathrm{H}))$ of blob/spot region is derived as 9.17 $\pm 0.08(1 \sigma)$. Using the relation between $\log (\mathrm{O} / \mathrm{H})$ and $\log (\mathrm{N} / \mathrm{O})$ (Figure 8 by Liang et al. $2006)$, the $\log (\mathrm{N} / \mathrm{O})$ abundance of blob/spot region is estimated to be $-0.50 \pm 0.13(1 \sigma)$. Here the scatter in $\mathrm{N} / \mathrm{O}$ to $\mathrm{O} / \mathrm{H}$ in Figure 8 by Liang et al. (2006) was estimated to be about $0.1 \mathrm{dex}(1 \sigma)$ by eye-inspection. When we use the Anders \& Grevesse (1989) abundance set, whose $\log (\mathrm{N} / \mathrm{O})$ ratio is -0.88 , the $\mathrm{N} / \mathrm{O}$ ratio of blob/spot region is about 2.4 times larger than the solar value. This value of 2.4 does not change even if we use the Grevesse et al. (2007) abundance set, because its N/O ratio is equal to that of the Anders \& Grevesse (1989) abundance set. This value is similar to that in the galactic wind (Section 4.1), although it is difficult to estimate the abundance precisely. To explain this similarity, it is plausible that the gas of the galactic wind is chemically evolved in starburst regions and then is blown out. 


\subsection{Extent of the galactic wind}

In $\mathrm{H}$ II region $\mathrm{B}$, three clumps are found in our $\mathrm{H} \alpha$ map. The northern and western clumps are on the spiral arm, while the southeastern one is not. Soft X-ray (0.3-2.0 keV) is detected around H II region B, including the three clumps (Figures 2 (h) and 3 (b)). Therefore, it is difficult to determine from only the $\mathrm{H} \alpha$ map whether the southeastern clump is a part of the galactic wind or an H II region on the galactic disk with an X-ray emitting gas accidentally located on the same line of sight. This issue is important when considering the structure of the galactic wind, i.e., whether or not the tip of the galactic wind is cooled down enough to emit the $\mathrm{H} \alpha$. Although $\mathrm{H}$ II region $\mathrm{B}$ was observed and considered to be

H II regions through a long-slit spectroscopy (McCarthy et al. 1987), the line ratio of each clump was not discussed. In our data, the southeastern clump, as well as the northern and the western ones, have the same $[\mathrm{N}$ II] $] / \mathrm{H} \alpha$ and $[\mathrm{S} \mathrm{II}] / \mathrm{H} \alpha$ ratios as $\mathrm{H}$ II regions in nearby disk galaxies (Moustakas \& Kennicutt 2006). Moreover, the equivalent widths of three clumps are similar: $\sim 40 \AA$ (Figure 2 (g)). Consequently, we consider that the three clumps of H II region $\mathrm{B}$ are not a part of the galactic wind but are $\mathrm{H}$ II regions on the galactic disk.

\subsection{Kinetic energy of the galactic wind}

We derive the properties of the galactic wind, given that the region where the $[\mathrm{N} \mathrm{II}] / \mathrm{H} \alpha$ ratio is greater than 1.0 (or the $[\mathrm{S} \mathrm{II}] / \mathrm{H} \alpha$ ratio is greater than 0.5 ) is the galactic wind (Veilleux et al. 2005; Bland-Hawthorn et al. 2007). First we estimate the H $\alpha$ luminosity of the wind. When the $[\mathrm{N} \mathrm{II}] / \mathrm{H} \alpha$ criterion is used, the $\mathrm{H} \alpha$ flux of the galactic wind is equal to $1.6 \times 10^{-13} \mathrm{erg} \mathrm{cm}^{-2} \mathrm{~s}^{-1}$, and its $\mathrm{H} \alpha$ luminosity is $2.3 \times 10^{38} \mathrm{erg} \mathrm{s}^{-1}$ when $3.5 \mathrm{Mpc}$ is used as a distance to NGC 253 (Rekola et al. 2005). When the [S II]/H $\alpha$ criterion is used instead, the $\mathrm{H} \alpha$ flux is equal to $8.9 \times 10^{-13} \mathrm{erg} \mathrm{cm}^{-2} \mathrm{~s}^{-1}$ and the $\mathrm{H} \alpha$ luminosity is $1.3 \times 10^{39}$ $\operatorname{erg~} \mathrm{s}^{-1}$. Hereafter, we adopt the $[\mathrm{S} \mathrm{II}] / \mathrm{H} \alpha$ criterion, because it is less affected by nitrogen enrichment (Section 4.1, 4.2) compared with the $[\mathrm{N} \mathrm{II}] / \mathrm{H} \alpha$ criterion. Because the total $\mathrm{H} \alpha+[\mathrm{N} \mathrm{II}] \lambda \lambda 6548,6583$ luminosity of NGC 253 is equal to $1.1 \times 10^{41} \mathrm{erg} \mathrm{s}^{-1}$ (Hoopes et al. 1996), the $\mathrm{H} \alpha$ luminosity of the galactic wind is only from $0.5 \%$ to $5 \%$ of the total luminosity. The actual fraction of the luminosity of the galactic wind may be even smaller, because extinction is not corrected in the above estimate although the central region of NGC 253 is heavily dusty (e.g., Sams et al. 1994). Secondly, we estimate the mass of ionized gas in the galactic wind. From the $[\mathrm{S}$ II $] \lambda 6716 /[\mathrm{S}$ II $] \lambda 6731$ ratio of 0.9 to 1.2 in the central region (Schulz \& Wegner 1992), the electron density is estimated to be from $100 \mathrm{~cm}^{-3}$ to $1000 \mathrm{~cm}^{-3}$ (Osterbrock \& Ferland 2006). Using the electron density of $100 n_{e, 2} \mathrm{~cm}^{-3}$, where $n_{e, 2}$ is normalized to $100 \mathrm{~cm}^{-3}$, and the recombination rate of $\mathrm{H} \alpha$ of $8.7 \times 10^{-14} \mathrm{~cm}^{3} \mathrm{~s}^{-1}$ 
(Osterbrock \& Ferland 2006), the $\mathrm{H} \alpha$ emitting gas mass of the galactic wind is estimated to be $8.6 \times 10^{4} n_{e, 2}^{-1} M_{\odot}$. We assumed that the northwest side of the galactic wind, which is not detected in our observations due to heavy extinction, actually exists as well as the southeast side of it (Section 3.1). For simplicity, we assumed the symmetric shape of the galactic wind in order to roughly estimate the volume filling factor, although it is non-axisymmetric about the galactic minor axis. The assumed shape of the galactic wind is a truncated cone structure whose opening angle is $30^{\circ}$ and diameter of the base and slant height are $500 \mathrm{pc}$ and 700 pc, respectively. These values are measured through the $\mathrm{H} \alpha$ map. Then, the volume filling factor of the galactic wind is estimated to be $4.2 \times 10^{-4} n_{e, 2}^{-2}$. The limb-brightening in the $\mathrm{H} \alpha$ and the X-ray (Section 3.1) and the small filling factor suggest that the galactic wind has a hollow structure. Finally, using the outflow velocity of $390 \mathrm{~km} \mathrm{~s}^{-1}$ (Schulz \& Wegner 1992), the kinetic energy of the galactic wind is estimated to be $1.3 \times 10^{53} n_{e, 2}^{-1} \mathrm{erg}$. The dynamical time scale $\tau_{d y n}$ derived from the length of the filament $\mathrm{B}(\sim 700 \mathrm{pc})$ and the outflow velocity $\left(390 \mathrm{~km} \mathrm{~s}^{-1}\right)$ is $\sim 1.8 \mathrm{Myr}$, and the kinetic energy is increased by $2.3 \times 10^{39} n_{e, 2}^{-1}$ erg s${ }^{-1}$ on average.

In order to consider whether the energy input from supernovae can supply the kinetic energy of the galactic wind, we estimate first the $\mathrm{H} \alpha$ luminosity of the bright blob, which is the brightest region in the central region of NGC 253. Because of heavy extinction in the central region of NGC 253, we have corrected the effect of extinction using the ratio of the $\mathrm{H} \alpha$ flux to the $\mathrm{Br} \gamma$ flux (Forbes et al. 1993). We assumed the intrinsic $\mathrm{Br} \gamma / \mathrm{H} \alpha$ flux ratio of $9.80 \times 10^{-3}$ (Osterbrock \& Ferland 2006) and the extinction law of $\mathrm{A}(6563 \AA)=0.79 \mathrm{~A}_{V}$ and $\mathrm{A}(2.2 \mu \mathrm{m})=0.15 \mathrm{~A}_{V}$ (Whitford 1958; Miller \& Mathews 1972). The $\mathrm{H} \alpha$ flux of the bright blob in $2^{\prime \prime}$ aperture, which is the same as used by Forbes et al. (1993), is $5.5 \times 10^{-14} \mathrm{erg} \mathrm{cm}^{-2} \mathrm{~s}^{-1}$, and $\mathrm{A}_{V}$ is estimated to $6.9 \mathrm{mag}$. The extinction-corrected $\mathrm{H} \alpha$ flux is $8.3 \times 10^{-12} \mathrm{erg} \mathrm{cm}^{-2} \mathrm{~s}^{-1}$, and the $\mathrm{H} \alpha$ luminosity is $1.2 \times 10^{40} \mathrm{erg} \mathrm{s}^{-1}$, which corresponds to the star formation rate of $0.096 M_{\odot} \mathrm{yr}^{-1}$ (Kennicutt 1998). When we assume that the star formation rate of the bright blob is constant, the supplied kinetic energy through supernovae and stellar winds from this region in $1.8 \mathrm{Myr}\left(=\tau_{\text {dyn }}\right)$ is estimated to be $5.6 \times 10^{53} \mathrm{erg}$ (Starburst99: Leitherer et al. 1999). We assumed Salpeter initial mass function (Salpeter 1955), $M_{u p}=100 M_{\odot}$ and $M_{l o w}=1 M_{\odot}$ as cut-off masses, metallicity $\mathrm{Z}=0.020$, and that the galactic wind began to blow when the first supernova occurred in the bright blob (3.6 Myr: Figure 114 in Leitherer et al. 1999). Therefore, the energy input from the bright blob is much greater than the kinetic energy of the galactic wind. This suggests that starbursts are the main energy source of the galactic wind in NGC 253.

We compare the properties of the galactic winds in M82 (Shopbell \& Bland-Hawthorn 1998), NGC 1482 (Veilleux \& Rupke 2002), and NGC 253, although the definition of galactic wind regions in M82 is quite different from that in NGC 253 and NGC 1482. Shopbell \& Bland-Hawthorn 
(1998) defined the galactic wind regions by simply excluding within approximately 8 "of the disk (+ halo component), while we and Veilleux \& Rupke (2002) used the criterion of the $[\mathrm{S} \mathrm{II}] / \mathrm{H} \alpha$ and $[\mathrm{N} \mathrm{II}] / \mathrm{H} \alpha$ ratio, respectively. The kinetic energy of the galactic wind in NGC $253\left(1.3 \times 10^{53} \mathrm{erg}\right)$ is found to be smaller than those in M82 $\left(2.1 \times 10^{55} \mathrm{erg}\right)$ and in NGC 1482 $\left(2 \times 10^{53} \mathrm{erg}\right)$. There are three possibilities for the difference in the energies of the galactic winds: the masses, the velocities, and the dynamical timescales. The mass in NGC 1482 is about 4.2 times, and the mass in M82 is about 67 times as large as that in NGC 253 $\left(8.6 \times 10^{4} M_{\odot}\right)$. The outflow velocities and the dynamical timescales do not vary drastically among these galaxies: $390 \mathrm{~km} \mathrm{~s}^{-1}$ and $1.8 \mathrm{Myr}$ in NGC 253, $250 \mathrm{~km} \mathrm{~s}^{-1}$ and $6 \mathrm{Myr}$ in NGC 1482 , and $600 \mathrm{~km} \mathrm{~s}^{-1}$ and $3.4 \mathrm{Myr}$ in M82, respectively. Therefore, the difference of the outflow mass in each galaxy, which may depend on the strength of starburst in the central region, probably dominantly makes the variety of the kinetic energy of the galactic wind, although the combination of the differences of the mass, the velocity, and the dynamical time scale will determine the exact value.

\section{SUMMARY}

We have observed the central region of NGC 253 with the Fabry-Perot mode of the Kyoto3DII mounted on the Subaru Telescope. We have presented the continuum and the $\mathrm{H} \alpha$ images, and the $[\mathrm{N} \mathrm{II}] \lambda 6583 / \mathrm{H} \alpha$, [S II] $\lambda \lambda 6716,6731 / \mathrm{H} \alpha$, and $[\mathrm{N}$ II] $\lambda 6583 /[\mathrm{S} \mathrm{II}] \lambda \lambda 6716,6731$ ratio maps. The $[\mathrm{N} \mathrm{II}] / \mathrm{H} \alpha$ of filament $\mathrm{B}$ is larger than 1.0, which indicates that this region is ionized/excited by shock. Non-axisymmetry in the galactic wind, which is also seen in M82, is found. The shapes of the galactic wind and the line ratio maps are non-axisymmetric about the galactic minor axis: e.g., the $[\mathrm{N} \mathrm{II}] / \mathrm{H} \alpha$ of filament $\mathrm{A}$ is smaller than that of filament B. The $[\mathrm{N} \mathrm{II}] / \mathrm{H} \alpha$ map enables us to isolate filament $\mathrm{C}$ from the galactic wind. Using the $[\mathrm{S} \mathrm{II}] / \mathrm{H} \alpha$ map, we extract the galactic wind region, and estimate the mass and the kinetic energy of the galactic wind: $8.6 \times 10^{4} M_{\odot}$ and $1.3 \times 10^{53} \mathrm{erg}$, respectively. The mass and the kinetic energy of the galactic wind in NGC 253 are smaller than those in M82 and NGC 1482. The filling factor of the galactic wind is much smaller than unity, which is consistent with that the galactic wind has a hollow structure. The energy input from supernovae in the bright blob can sufficiently supply the energy of the galactic wind. From the [N II]/[S II] ratio map, the interstellar gas in the central region and in the galactic wind is found to have a high nitrogen enrichment. The bright blob and spot $\mathrm{i}$, which are stellar clusters found in $H S T$ observations, have especially high [N II]/[S II] ratios due to heavy nitrogen enrichment.

We thank the staff at the Subaru Telescope for their help during the test observing run. We also thank T. Ohtani, T. Hayashi, M. Ishii, Y. Okita, A. Akita, J. Sudo, M. Sasaki, N. 
Takeyama, M. A. Malkan, and A. R. Jenner for discussions. This work was supported by the Grant-in-Aid for the Global COE Program "The Next Generation of Physics, Spun from

Universality and Emergence" from the Ministry of Education, Culture, Sports, Science and Technology (MEXT) of Japan.

\section{REFERENCES}

Anders, E., \& Grevesse, N. 1989, Geochim. Cosmochim. Acta, 53, 197

Athanassoula, E. 1992, MNRAS, 259, 328

Binney, J., Gerhard, O. E., Stark, A. A., Bally, J., \& Uchida, K. I. 1991, MNRAS, 252, 210

Bland, J., \& Tully, R. B. 1989, AJ, 98, 723

Bland-Hawthorn, J., Veilleux, S., \& Cecil, G. 2007, Ap\&SS, 311, 87

Cooper, J. L., Bicknell, G. V., Sutherland, R. S., \& Bland-Hawthorn, J. 2008, ApJ, 674, 157

Dopita, M. A., \& Sutherland, R. S. 1995, ApJ, 455, 468

Ferrara, A., \& Tolstoy, E. 2000, MNRAS, 313, 291

Forbes, D. A., Ward, M. J., Rotaciuc, V., Blietz, M., Genzel, R., Drapatz, S., van der Werf, P. P., \& Krabbe, A. 1993, ApJ, 406, L11

Grevesse, N., Asplund, M., \& Sauval, A. J. 2007, Space Science Reviews, 130, 105

Hoopes, C. G., Walterbos, R. A. M., \& Greenwalt, B. E. 1996, AJ, 112, 1429

Kauffmann, G., et al. 2003, MNRAS, 346, 1055

Kennicutt, R. C., Jr. 1998, ARA\&A, 36, 189

Kewley, L. J., Dopita, M. A., Sutherland, R. S., Heisler, C. A., \& Trevena, J. 2001, ApJ, 556,121

Kobayashi, M. A. R., Totani, T., \& Nagashima, M. 2007, ApJ, 670, 919

Leitherer, C., et al. 1999, ApJS, 123, 3

Liang, Y. C., Yin, S. Y., Hammer, F., Deng, L. C., Flores, H., \& Zhang, B. 2006, ApJ, 652, 257 
McCarthy, P. J., van Breugel, W., \& Heckman, T. 1987, AJ, 93, 264

Miller, J. S., \& Mathews, W. G. 1972, ApJ, 172, 593

Moustakas, J., \& Kennicutt, R. C., Jr. 2006, ApJ, 651, 155

Osterbrock, D. E., \& Ferland, G. J. 2006, Astrophysics of gaseous nebulae and active galactic nuclei, 2nd. ed. by D.E. Osterbrock and G.J. Ferland. Sausalito, CA: University Science Books, 2006

Pagel, B. E. J. 1997, Nucleosynthesis and Chemical Evolution of Galaxies, by Bernard E. J. Pagel, pp. 392. ISBN 0521550610. Cambridge, UK: Cambridge University Press, October 1997

Paglione, T. A. D., Yam, O., Tosaki, T., \& Jackson, J. M. 2004, ApJ, 611, 835

Pence, W. D. 1980, ApJ, 239, 54

Peng, R., Zhou, S., Whiteoak, J. B., Lo, K. Y., \& Sutton, E. C. 1996, ApJ, 470, 821

Rekola, R., Richer, M. G., McCall, M. L., Valtonen, M. J., Kotilainen, J. K., \& Flynn, C. 2005, MNRAS, 361, 330

Sakamoto, K., et al. 2006, ApJ, 636, 685

Salpeter, E. E. 1955, ApJ, 121, 161

Sams, B. J., III, Genzel, R., Eckart, A., Tacconi-Garman, L., \& Hofmann, R. 1994, ApJ, 430, L33

Sato, T., Martin, C. L., Noeske, K. G., Koo, D. C., \& Lotz, J. M. 2008, ArXiv e-prints, 804, arXiv:0804.4312

Schulz, H., \& Wegner, G. 1992, A\&A, 266, 167

Shapley, A. E., Steidel, C. C., Pettini, M., \& Adelberger, K. L. 2003, ApJ, 588, 65

Shopbell, P. L., \& Bland-Hawthorn, J. 1998, ApJ, 493, 129

Strickland, D. K., Heckman, T. M., Weaver, K. A., \& Dahlem, M. 2000, AJ, 120, 2965

Strickland, D. K., Heckman, T. M., Colbert, E. J. M., Hoopes, C. G., \& Weaver, K. A. 2004, ApJ, 606, 829

Sugai, H., et al. 2000, Proc. SPIE, 4008, 558 
Sugai, H., Ozaki, S., Hattori, T., \& Kawai, A. 2002, Galaxies: the Third Dimension, 282, 433

Sugai, H., Davies, R. I., \& Ward, M. J. 2003, ApJ, 584, L9

Sugai, H., et al. 2004, Proc. SPIE, 5492, 651

Sugai, H., et al. 2004, ApJ, 615, L89

Tapken, C., Appenzeller, I., Noll, S., Richling, S., Heidt, J., Meinköhn, E., \& Mehlert, D. 2007, A\&A, 467, 63

Tüllmann, R., Pietsch, W., Rossa, J., Breitschwerdt, D., \& Dettmar, R.-J. 2006, A\&A, 448, 43

Turner, J. L., \& Ho, P. T. P. 1985, ApJ, 299, L77

Ulvestad, J. S., \& Antonucci, R. R. J. 1997, ApJ, 488, 621

Veilleux, S., \& Rupke, D. S. 2002, ApJ, 565, L63

Veilleux, S., Cecil, G., \& Bland-Hawthorn, J. 2005, ARA\&A, 43, 769

Watson, A. M., et al. 1996, AJ, 112, 534

Whitford, A. E. 1958, AJ, 63, 201 

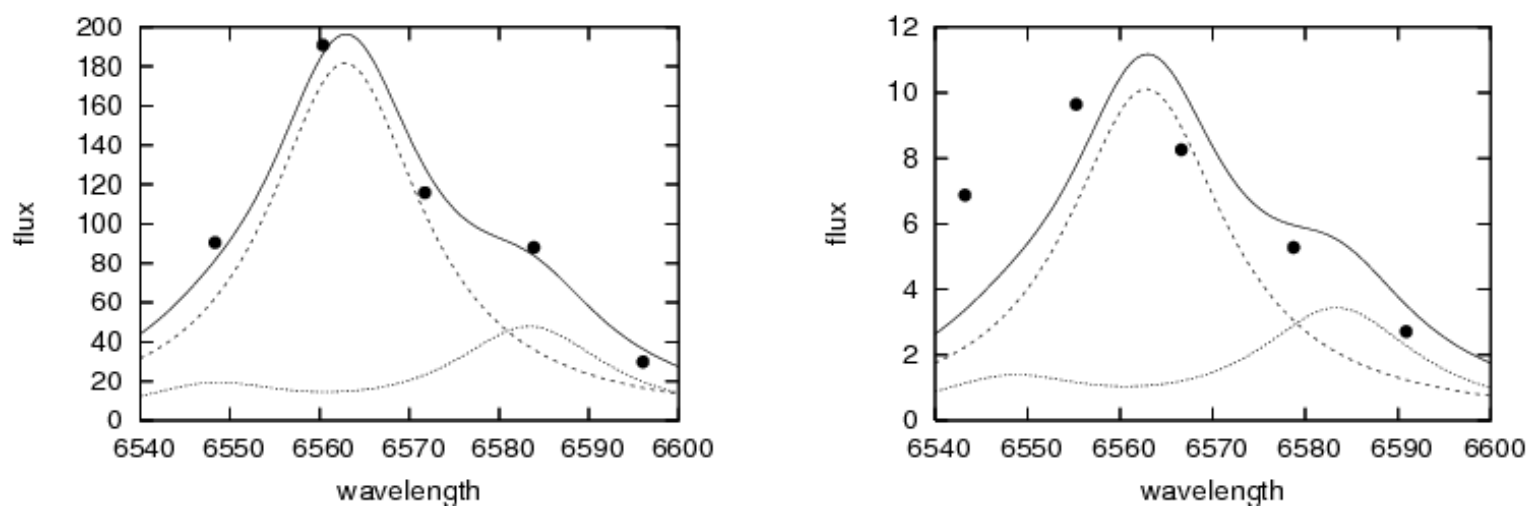

Fig. 1.- Good (left panel) and poor examples (right panel) of fitting of the $\mathrm{H} \alpha+[\mathrm{N} \mathrm{II}]$ set. The flux is shown as a function of wavelength in units of $\AA$. The observed values are plotted in filled circles. The dashed line and dotted line represent the fluxes from the $\mathrm{H} \alpha$ and the $[\mathrm{N} \mathrm{II}] \lambda \lambda 6548,6583$, respectively, as a function of the central wavelength of the transmission curve of etalon. The solid line is sum of the dashed line and dotted line. 

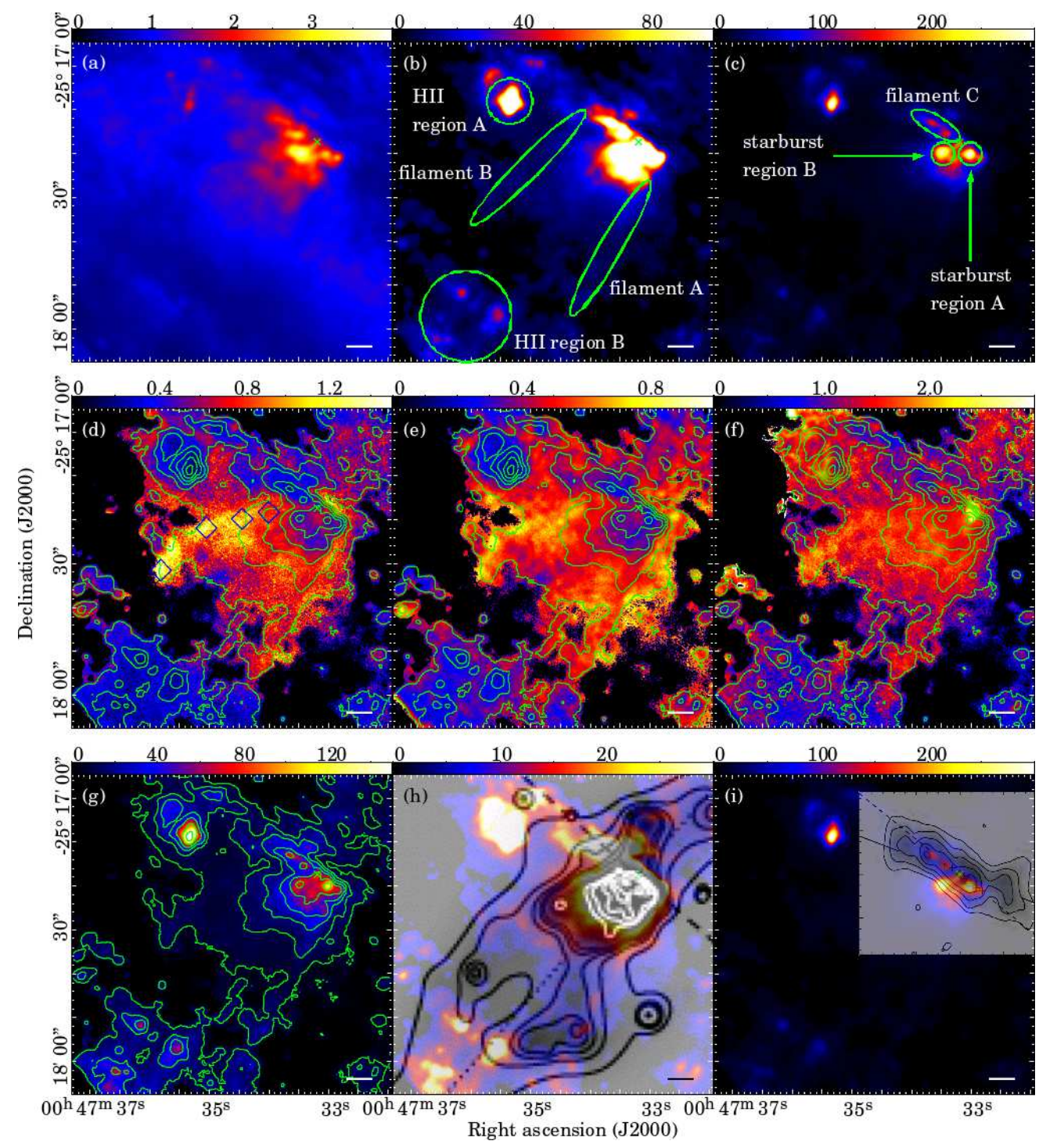

Fig. 2.- The continuum and $\mathrm{H} \alpha$ image, and the line ratio maps of the central region of NGC 253. North is up and east is to the left. The green cross at the center of each figure represents the center of NGC 253 derived from the radio continuum (Ulvestad \& Antonucci 1997). The bars in all figures at lower left represent the length of 100 pc. (a) The continuum image in units of $10^{-16} \mathrm{erg} \mathrm{cm}^{-2} \mathrm{~s}^{-1} \operatorname{arcsec}^{-2} \AA^{-1}$. (b) The deep and (c) shallow H $\alpha$ image in units of $10^{-16} \mathrm{erg}^{-2} \mathrm{~s}^{-1} \operatorname{arcsec}^{-2}$. (d) The $[\mathrm{N} \mathrm{II}] / \mathrm{H} \alpha$ line ratio map. The background noise level in the $\mathrm{H} \alpha$ flux $(1 \sigma)$ is $1.9 \times 10^{-16} \mathrm{erg} \mathrm{cm}^{-2} \mathrm{~s}^{-1} \operatorname{arcsec}^{-1}$. The green contours are $4 \sigma, 8 \sigma, 16 \sigma, 32 \sigma, 64 \sigma$, and $128 \sigma$ of the $\mathrm{H} \alpha$ flux. The region where the $\mathrm{H} \alpha$ flux is weaker than $3 \sigma\left(=5.7 \times 10^{-16} \mathrm{erg} \mathrm{cm}^{-2} \mathrm{~s}^{-1} \operatorname{arcsec}^{-2}\right)$ is shown in black. The blue squares represent the regions where the $[\mathrm{N} \mathrm{II}] / \mathrm{H} \alpha$ and $[\mathrm{S} \mathrm{II}] / \mathrm{H} \alpha$ ratios are measured and are plotted with green squares in Figure 5. (e) The [S II] $/ \mathrm{H} \alpha$ line ratio map. (f) The [N II]/[S II] line ratio map. (g) The $\mathrm{H} \alpha$ equivalent width map in units of $\AA$. (h) The continuum subtracted $\mathrm{H} \alpha$ image in units of $10^{-16} \mathrm{erg} \mathrm{cm}^{-2} \mathrm{~s}^{-1} \operatorname{arcsec}^{-2}$. The contours show the intensity of soft X-ray (0.3-2.0 $\mathrm{keV}$ : Strickland et al. 2000). (i) The continuum subtracted $\mathrm{H} \alpha$ image in units of $10^{-16}$ erg $\mathrm{cm}^{-2} \mathrm{~s}^{-1} \operatorname{arcsec}^{-2}$. The contours show the intensity of CO J $=1 \rightarrow 0$ (Paglione et al. 2004). 


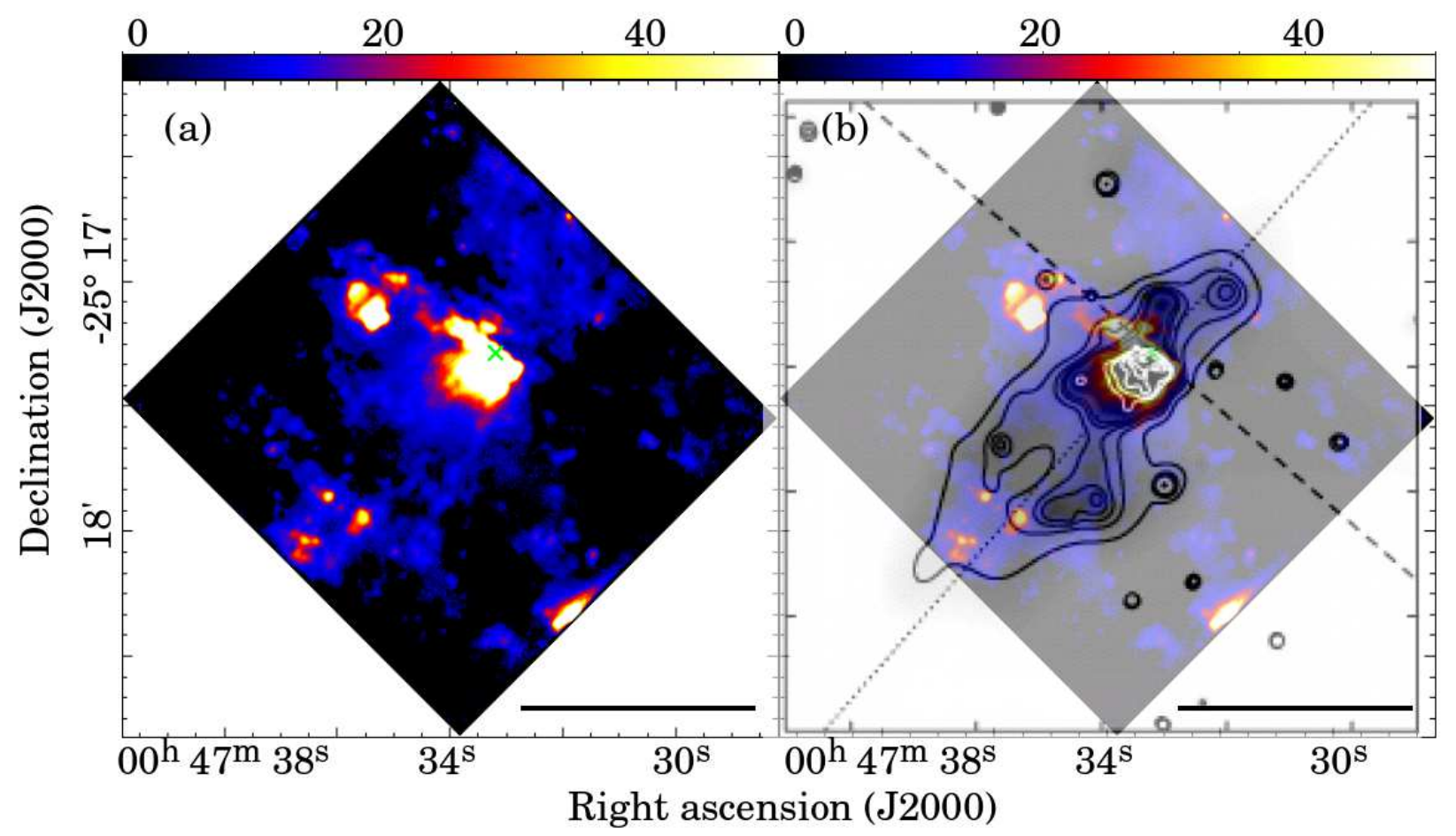

Fig. 3.- (a) The continuum subtracted H $\alpha$ images of NGC 253 in units of $\mathrm{erg} \mathrm{cm}^{-2} \mathrm{~s}^{-1}$ $\operatorname{arcsec}^{-2}$. North is up and east is to the left. The green cross at the center of this figure represents the center of NGC 253 derived from the radio continuum (Ulvestad \& Antonucci 1997). The bar at lower left represents the length of $1 \mathrm{kpc}$. The region where the $\mathrm{H} \alpha$ flux is weaker than $3 \sigma\left(=5.7 \times 10^{-16} \mathrm{erg} \mathrm{cm}^{-2} \mathrm{~s}^{-1} \operatorname{arcsec}^{-2}\right)$ is shown in black. The bright point at the lower right edge is a ghost of the central region. (b) The continuum subtracted $\mathrm{H} \alpha$ images of NGC 253 in units of erg $\mathrm{cm}^{-2} \mathrm{~s}^{-1} \operatorname{arcsec}^{-2}$. The contours show the intensity of soft X-ray (0.3-2.0 keV: Strickland et al. 2000). 


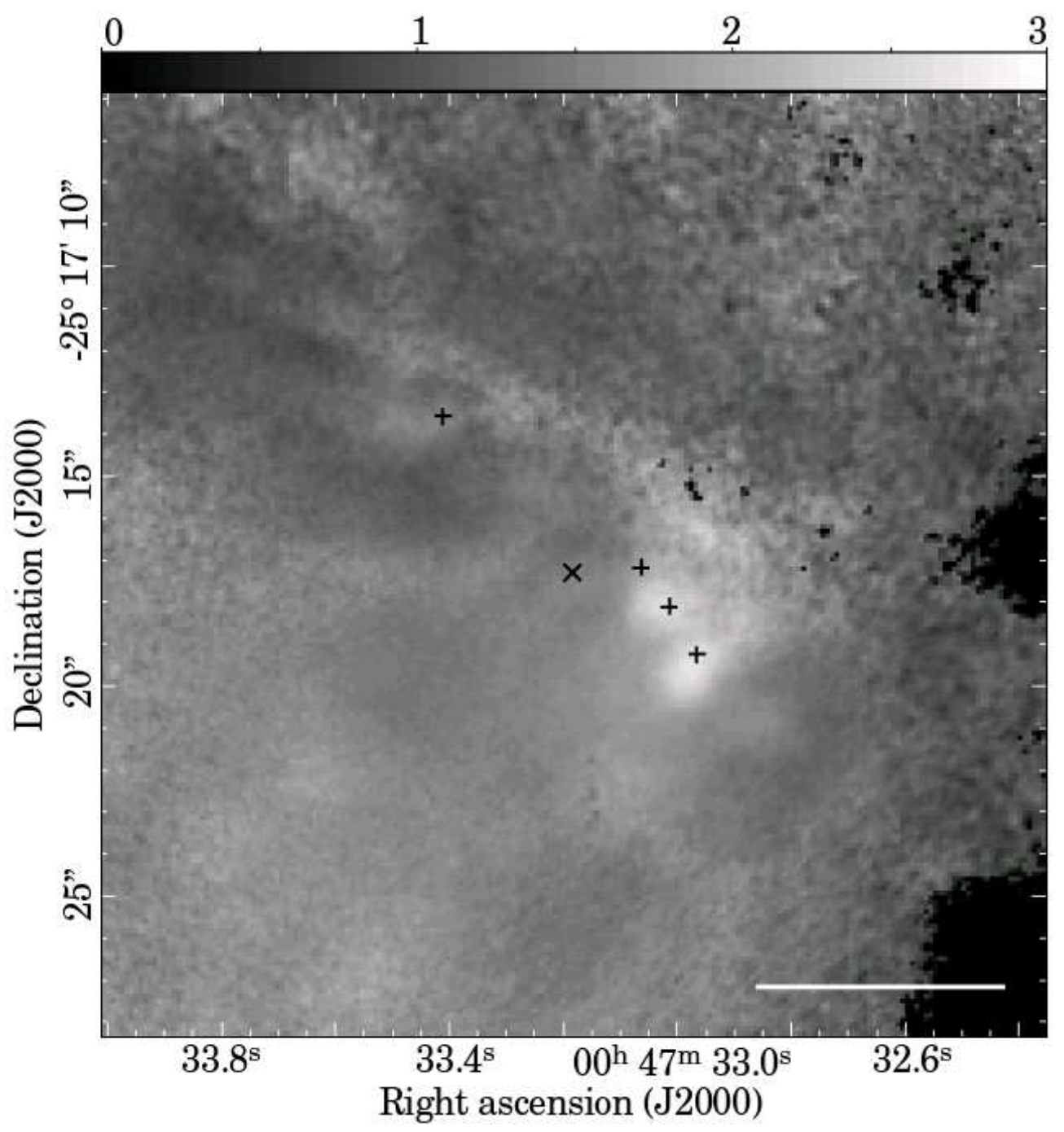

Fig. 4.- The $[\mathrm{N} \mathrm{II}] \lambda 6583 /[\mathrm{S}$ II $] \lambda \lambda 6716,6731$ ratio map in the central region. North is up and east is to the left. The cross at the center of this figure represents the center of NGC 253 derived from the radio continuum (Ulvestad \& Antonucci 1997). The bar at lower left represents the length of 100 pc. Four plus signs represent the positions of star clusters found by $H S T$, which are spot a, spot $\mathrm{n}$, spot $\mathrm{i}$, and the bright blob starting from the left. The region where the $\mathrm{H} \alpha$ flux is weaker than $3 \sigma\left(=5.7 \times 10^{-16} \mathrm{erg} \mathrm{cm}^{-2} \mathrm{~s}^{-1} \operatorname{arcsec}^{-2}\right)$ is shown in black. [See the electronic edition of the Journal for a color version of this figure.] 


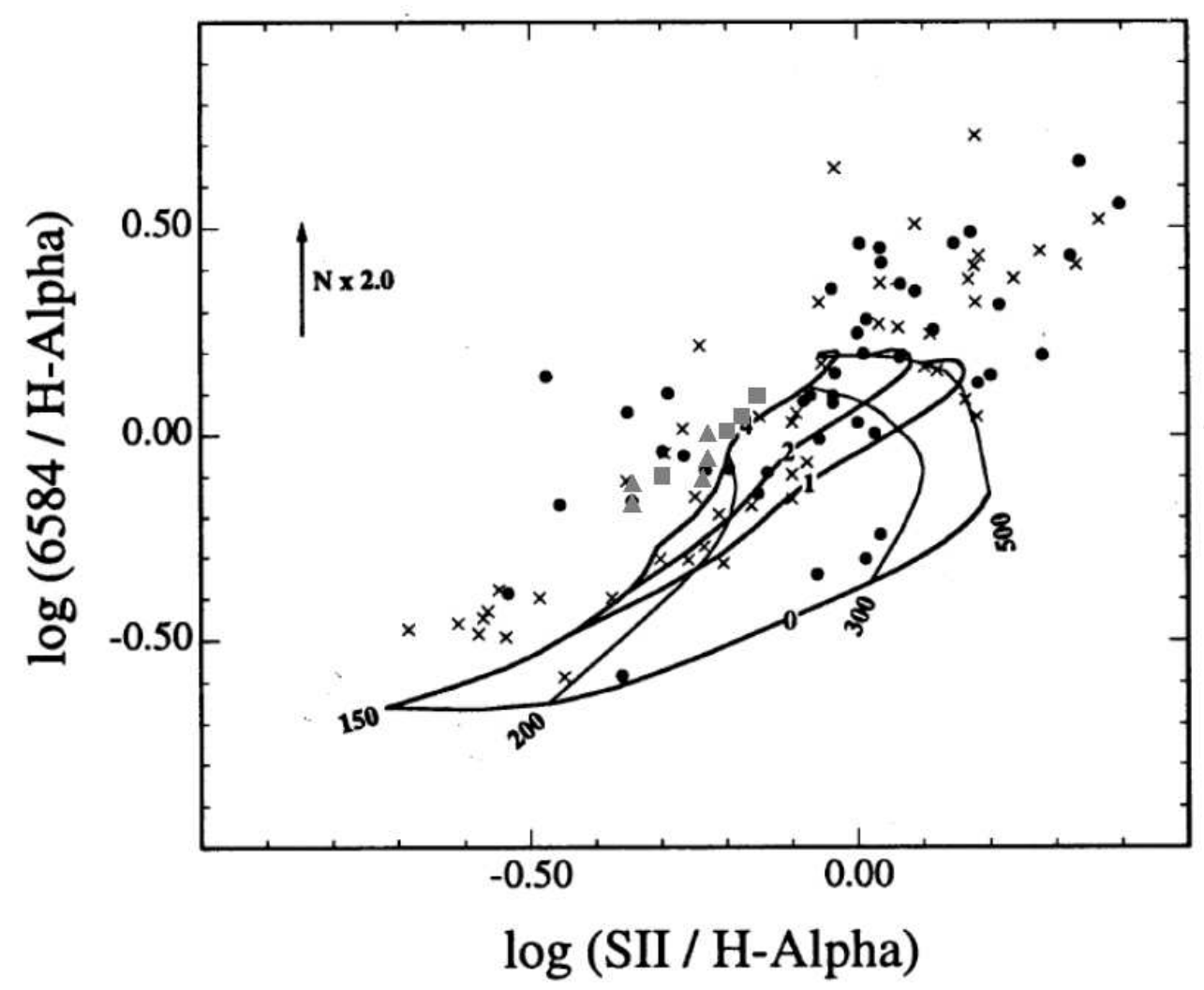

Fig. 5.- The $[\mathrm{N} \mathrm{II}] / \mathrm{H} \alpha$ ratio vs the $[\mathrm{S} \mathrm{II}] / \mathrm{H} \alpha$ ratio diagram of the shock model (shockonly: Dopita \& Sutherland 1995). The numbers below the model curves represent the shock velocities in units of $\mathrm{km} \mathrm{s}^{-1}$, and the numbers on the curves represent the magnetic parameters $\left(B / n^{1 / 2}\right)$ in units of $\mu \mathrm{G} \mathrm{cm}{ }^{3 / 2}$. The effect of a factor of 2 increase in the $\mathrm{N} / \mathrm{O}$ ratio is shown by the arrow. Filled circles are for LINERs while crosses are for radio galaxies (Dopita \& Sutherland 1995). Four squares represent the line ratios observed at filament B in NGC 253 (see Figure 2 (d) for their positions) while five triangles represent those in typical regions of the galactic wind. The square drawn furthest left shows the line ratios closest to the galactic disk, with each subsequent square to the right lying at increasing distance. [See the electronic edition of the Journal for a color version of this figure.] 
Table 1. Fluxes, star formation rates, and line ratios of each region

\begin{tabular}{cccccccc}
\hline \hline Region & Aperture & $\mathrm{Flux}(\mathrm{H} \alpha)^{\mathrm{a}}$ & $\mathrm{SFR}^{\mathrm{b}}$ & $\mathrm{EW}(\mathrm{H} \alpha)^{\mathrm{c}}$ & {$[\mathrm{N} \mathrm{II}] / \mathrm{H} \alpha^{\mathrm{d}}$} & {$[\mathrm{S} \mathrm{II}] / \mathrm{H} \alpha^{\mathrm{e}}$} & {$[\mathrm{N}$ II $] /[\mathrm{S} \mathrm{II}]^{\mathrm{d}, \mathrm{e}}$} \\
\hline starburst region A & $3 " .5$ & 1.9 & 2.2 & 88 & 0.54 & 0.28 & 1.93 \\
starburst region B & $4 " .0$ & 2.5 & 2.9 & 64 & 0.51 & 0.35 & 1.45 \\
filament C & $11^{\prime \prime} .54 \times 3 " .47$ & 3.0 & 3.5 & 42 & 0.38 & 0.32 & 1.16 \\
H II region A & $6 " .0$ & 3.5 & 4.0 & $95(482)$ & 0.25 & 0.17 & 1.45 \\
H II region B(N) & $4 " .0$ & 0.34 & 0.39 & $36(316)$ & 0.24 & 0.22 & 1.06 \\
H II region B(W) & $6 " .0$ & 0.72 & 0.83 & $36(371)$ & 0.26 & 0.24 & 1.09 \\
H II region B(SE) & $8 " .0$ & 1.1 & 1.3 & $39(282)$ & 0.26 & 0.26 & 0.98 \\
\hline
\end{tabular}

Note. - The errors for flux $(\mathrm{H} \alpha), \mathrm{EW}(\mathrm{H} \alpha),[\mathrm{N} \mathrm{II}] / \mathrm{H} \alpha,[\mathrm{S} \mathrm{II}] / \mathrm{H} \alpha$, and $[\mathrm{N} \mathrm{II}] /[\mathrm{S} \mathrm{II}]$ are less than $30 \%$, a factor of $2,30 \%, 10 \%$, and $30 \%$, respectively.

a The unit of flux is $10^{-13} \mathrm{erg}^{-2} \mathrm{sm}^{-1}$.

${ }^{\mathrm{b}}$ The star formation rates are estimated from the H $\alpha$ fluxes using the relation obtained by Kennicutt (1998). The unit of star formation rate is $10^{-3} M_{\odot} \mathrm{yr}^{-1}$.

${ }^{\text {c }}$ The unit of equivalent width is $\AA$. The value shown in parentheses is the equivalent width derived by subtracting the underlying diffuse continuum from the continuum emission.

$\mathrm{d}[\mathrm{N}$ II] means the $[\mathrm{N} \mathrm{II}] \lambda 6583$ line.

e $[\mathrm{S} \mathrm{II}]$ means the $[\mathrm{S} \mathrm{II}] \lambda \lambda 6716,6731$ lines. 